\title{
LOS ESTUDIANTES DE LA UNIVERSIDAD DE SANTIAGO DE COMPOSTELA ANTE LA DIVERSIDAD FUNCIONAL DE SUS COMPAÑEROS Y COMPAÑERAS
}

\author{
M. ${ }^{a}$ Emma Mayo Pais \\ Universidad de Santiago de Compostela
}

\begin{abstract}
RESUMEN: Para dar continuidad a investigaciones previas, la presente investigación se plantea con el objetivo general de conocer las ideas y actitudes de los estudiantes de la Universidad de Santiago de Compostela (USC) hacia sus iguales con diversidad funcional (discapacidad). El método de recogida de información seleccionado ha sido la encuesta, utilizando un cuestionario como instrumento para la recopilación de datos. Participaron un total de 400 estudiantes sin discapacidad de la USC, que cursan sus estudios en las diferentes áreas de conocimiento. Los resultados indican que los estudiantes de la USC manifiestan unas ideas y actitudes positivas, de aceptación hacia la integración en la universidad de sus compañeros y compañeras con diversidad funcional. A diferencia de lo que ocurre en otras investigaciones, la edad, el género y el curso académico en el que están matriculados no introdujeron diferencias estadísticamente significativas en cuanto a las ideas y actitudes manifestadas.
\end{abstract}

PALABRAS CLAVE: universidad, integración, discapacidad, inclusión educativa.

\section{STUDENTS OF THE UNIVERSITY OF SANTIAGO DE COMPOSTELA IN THE FACE OF FUNCTIONAL DIVERSITY OF THEIR PEERS}

\footnotetext{
ABSTRACT: To continue previous research, this research is proposed in order to know the ideas and attitudes of students of the University of Santiago de Compostela (USC) towards their peers with functional diversity (disability). The method selected for collecting information has been the survey, using a questionnaire as a tool for data collection. A total of 400 non-disabled students from the USC participated, studying in the different areas of knowledge. The results indicate that USC students express positive ideas and attitudes, of acceptance towards the integration into the university of their peers with
} 
functional diversity. Unlike other research, the age, gender and academic year in which they are enrolled did not introduce statistically significant differences in the ideas and attitudes expressed.

KEYWORDS: University, integration, disability, inclusive education.

Recibido: $28 / 03 / 2020$

Aceptado: 29/06/2020

Correspondencia: M. ${ }^{a}$ Emma Mayo Pais, Departamento de Psicología Evolutiva y de la Educación, Facultad de Psicología, Universidad de Santiago de Compostela, Rúa Xosé María Suárez Núñez, s/n. Campus Vida, 15782 Santiago de Compostela (España). Email: emma.mayo@usc.es

\section{INTRODUCCIÓN}

El sistema educativo juega un papel importante en la inclusión social de las personas con diversidad funcional (discapacidad). No obstante, la apuesta por una educación inclusiva llega a la educación superior con retraso respecto a los niveles educativos obligatorios (Fundación ONCE, 2014; Moriña, 2017), pues a nivel universitario no es hasta el año 2001 cuando la Ley Orgánica de Universidades (LOU) (posteriormente modificada por la Ley 4/2007, de 12 de abril, LOMLOU) recoge la inclusión de las personas con discapacidad en las universidades, contemplando los principios de igualdad de oportunidades, no discriminación y accesibilidad universal de las personas con discapacidad. Pese a ello, el impulso real ha venido con la construcción del Espacio Europeo de Educación Superior (EEES), al asumir el compromiso con la creación de entornos de formación accesibles a todas las personas (Rodríguez-Martín y Álvarez-Arregui, 2014).

En el año 2006 la ONU firma la Convención sobre los derechos de las personas con discapacidad, en la que se habla del derecho a la educación en un sistema educativo inclusivo, de calidad y gratuito. A pesar de la firma y ratificación de esta Convención, parece que todavía queda mucho por hacer, especialmente en las universidades. En este ámbito, el colectivo de personas con diversidad funcional no representa más que el 1.3 por ciento de la población universitaria (Fundación Universia, 2014), situándose en el 1.2 por ciento en estudios de postgrado y máster, y en el 0.6 por ciento en estudios de doctorado (Huete, 2017). En cuanto a la población general con estudios superiores, solo representan el 6.7 por ciento (Jiménez y Huete, 2016). Además, los estudiantes con discapacidad acceden a los estudios universitarios, mayoritariamente, en las universidades abiertas o a distancia (Lagoke, Komolafe, Ige y Oladejo, 2010; Nabeef, 2009; Richarson, 2010), lo que dificulta las relaciones entre pares (McAndrew, Farrow y Cooper, 2012), uno de los aspectos cruciales para mejorar las actitudes hacia la discapacidad, conseguir un mayor grado de sensibilización y lograr la inclusión del alumnado con diversidad funcional (NovoCorti y Muñoz-Cantero, 2012). 
Dado que la universidad inclusiva no sólo favorece a las personas con discapacidad, sino a todos los estudiantes en general (Alonso, Navarro y Vicente, 2008; Esteban-Guitart, Rivas y Pérez, 2012 y Moriña, 2008), se hace necesario, tal y como sostiene la UNESCO (2016), "garantizar una educación inclusiva y equitativa de calidad y promover oportunidades de aprendizaje permanente para todos" (Objetivo de Desarrollo Sostenible 4) y "asegurar el acceso en condiciones de igualdad para todos los hombres y las mujeres a una formación técnica, profesional y superior de calidad, incluida la enseñanza universitaria" (Meta 4.3.), pues "ninguna meta educativa debería considerarse lograda a menos que se haya logrado para todos" (Preámbulo, punto 7).

Según varios autores (Bueno, 2010; Flórez, Aguado y Alcedo, 2009; Hughes et al., 2001; Krahé y Altwasser, 2006; Luque, Carrión y Fernández, 2017 y Solesvik, Westhead, Kolvereid y Matlay, 2012), los avances hacia la inclusión universitaria pasan, entre otros, por conocer las ideas y actitudes que manifiestan los estudiantes universitarios hacia sus iguales con diversidad funcional, puesto que éstas influirán en el nivel de inclusión de los mismos.

Se han realizado ya algunos estudios al respecto tanto a nivel nacional, en la Universidad de Almería (Sánchez-Palomino, 2009) o en la Universidad de Burgos (Martínez, 2010), como a nivel europeo, en la Universidad de Bolonia -Italia- (Luque de la Rosa y Gutiérrez-Cáceres, 2013) y en la Universidad de Minho -Portugal- (Hernández-Garre, Fernández-Martínez, Carrión-Martínez y Avilés-Soler, 2019), alcanzando también interés el tema a nivel internacional con el estudio de Guzmán-Zamora y Sánchez-Palomino (2011), en el Estado de Plaxcala, (México).

Con el fin de aportar nuevos datos al respecto, la presente investigación se formula con el objetivo de conocer las ideas y actitudes de los estudiantes de la Universidad de Santiago de Compostela (USC) hacia sus compañeros con diversidad funcional y comparar si existen diferencias en las ideas y actitudes en función de la edad, el género, el área de conocimiento, el curso en el que se encuentran matriculados y/o la información que hayan podido recibir en la USC acerca de la presencia de compañeros/as con discapacidad.

\section{Método}

\subsection{Participantes}

Participaron en la investigación un total de 400 estudiantes sin discapacidad de la Universidad de Santiago de Compostela (USC). Para la selección de la muestra se Ilevó a cabo un muestreo aleatorio estratificado, por áreas de conocimiento y para estudiantes del primer al último curso de carrera, estableciéndose un nivel de confianza del $95 \%$ y un error muestral del 5\%. Cabe destacar que, si bien se debía recoger información de un total de 378 estudiantes para que la muestra fuese representativa, este número se ha redondeado a 400, siguiendo la distribución normativa por áreas de conocimiento (ver Tabla 1). 
Tabla 1. Frecuencia por área de conocimiento, titulación y curso

\begin{tabular}{|c|c|c|c|c|c|c|c|c|}
\hline \multirow{2}{*}{ ÁREA } & \multirow{2}{*}{ TITULACIÓN } & \multicolumn{6}{|c|}{ CURSO } & \multirow{2}{*}{ TOTAL } \\
\hline & & $1^{\circ}$ & $2^{a}$ & $3^{a}$ & $4^{a}$ & $5^{a}$ & $6^{\circ}$ & \\
\hline \multirow{3}{*}{$\begin{array}{l}\text { Ciencias Jurídicas y } \\
\text { Sociales }\end{array}$} & Licenciatura en Psicología & --- & --- & 3 & 86 & 11 & --- & 100 \\
\hline & $\begin{array}{l}\text { Grado en Educación Infan- } \\
\text { til }\end{array}$ & 63 & --- & --- & ---- & --- & --- & 63 \\
\hline & $\begin{array}{l}\text { Grado en Educación Pri- } \\
\text { maria }\end{array}$ & --- & 38 & 4 & ---- & --- & --- & 42 \\
\hline Humanidades & $\begin{array}{l}\text { Grado en Lengua y Litera- } \\
\text { tura Españolas }\end{array}$ & --- & 35 & 5 & ---- & --- & --- & 40 \\
\hline \multirow{2}{*}{ Ciencias de la Salud } & Licenciatura en Farmacia & --- & 17 & 17 & 6 & 19 & --- & 59 \\
\hline & Licenciatura en Medicina & -- & -- & -- & --- & 4 & 15 & 19 \\
\hline Enseñanzas Técnicas & $\begin{array}{l}\text { Grado/Licenciatura en In- } \\
\text { geniería Informática }\end{array}$ & 17 & 1 & 18 & --- & --- & --- & 36 \\
\hline $\begin{array}{l}\text { Ciencias } \\
\text { Experimentales }\end{array}$ & $\begin{array}{l}\text { Grado/Licenciatura en Fí- } \\
\text { sica }\end{array}$ & 12 & --- & 2 & 15 & 12 & --- & 41 \\
\hline \multicolumn{2}{|l|}{ TOTAL } & 92 & 91 & 49 & 107 & 46 & 15 & 400 \\
\hline
\end{tabular}

Del total de los participantes 278 eran mujeres (69.5\%) y 122 hombres $(30.5 \%)$. La media de edad fue de 21.82 años (SD 3.47), con un rango que va desde los 18 a los 45. El 80.69\% Ilevaban sus estudios a curso por año académico.

Se ha encontrado una relación estadísticamente significativa entre género y área de conocimiento en la que cursan sus estudios, de modo que ser hombre se asocia con cursar estudios en áreas de Ciencias Experimentales y Enseñanzas Técnicas, mientras que ser mujer se asocia a cursar estudios en áreas de Ciencias de la Salud, Ciencias Jurídicas y Sociales y Humanidades $\left[\chi^{2}(4)=169.91, p \leq .001\right]$ (ver Tabla 2).

Tabla 2. Género-Área de conocimiento

\begin{tabular}{|l|c|c|c|c|c|c|}
\hline Género & $\begin{array}{c}\text { Ciencias } \\
\text { de la } \\
\text { Salud }\end{array}$ & $\begin{array}{c}\text { Ciencias } \\
\text { Experimentales }\end{array}$ & $\begin{array}{c}\text { Ciencias } \\
\text { Jurídicas y } \\
\text { Sociales }\end{array}$ & $\begin{array}{c}\text { Enseñanzas } \\
\text { técnicas }\end{array}$ & Humanidades & TOTAL \\
\hline HOMBRE & 13 & 35 & 27 & 35 & 12 & $\mathbf{1 2 2}$ \\
\hline MUJER & 65 & 6 & 178 & 1 & 28 & $\mathbf{2 7 8}$ \\
\hline TOTAL & $\mathbf{7 8}$ & $\mathbf{4 1}$ & $\mathbf{2 0 5}$ & $\mathbf{3 6}$ & $\mathbf{4 0}$ & $\mathbf{4 0 0}$ \\
\hline
\end{tabular}

Solo 6 de los 400 participantes (1.5\%) han manifestado haber recibido algún tipo de información, en la USC, acerca de la presencia de compañeros/as con discapacidad en sus clases, siendo esta información proporcionada "por los propios compañeros con discapacidad" (0.5\%) o "por profesores de diversas materias" (1.0\%). 


\subsection{Instrumentos}

El método de recogida de información seleccionado ha sido la encuesta, utilizando un cuestionario como instrumento para la recopilación de datos (Bernal, 2018). Dicho cuestionario se encuentra dividido en dos partes:

- Primera parte: datos sociodemográficos básicos. Consta de un total de 8 ítems, que recogen información sobre: edad, género, titulación, curso realizado en la actualidad, facultad, si tienen o no algún tipo de discapacidad y, en caso de respuesta positiva, de qué tipo y si han recibido (en la USC) algún tipo de información o no acerca de la presencia de compañeros o compañeras con discapacidad.

- Segunda parte: ideas y actitudes de los estudiantes universitarios hacia sus iguales con diversidad funcional. Cuestionario específico, elaborado por Sánchez-Palomino (2009, pp. 173-175), dirigido a analizar las ideas y actitudes que sobre capacidades, formación y desarrollo profesional tienen los estudiantes de la Universidad de Almería (España) hacia el colectivo de estudiantes con necesidades especiales asociadas a una discapacidad. Consiste en una escala tipo Likert, compuesta por 32 ítems, con cuatro alternativas de respuesta (1. Totalmente en desacuerdo. 2. En desacuerdo. 3. De acuerdo. 4. Totalmente de acuerdo). Todas las preguntas son cerradas, a excepción de la última que aparece abierta con la formulación "observaciones que desea formular".

Tras realizar un pequeño estudio piloto, con el fin de garantizar la comprensión de las preguntas, se modificó ligeramente la estructura original de la segunda parte del cuestionario, agregando un nuevo ítem (ítem 4: los estudiantes con discapacidad necesitan más años para terminar una carrera) e incorporando una nueva alternativa de respuesta (3. Indiferente, neutro). La estructura final del cuestionario puede verse a continuación (ver Tabla 3).

Tabla 3. Estructura del cuestionario según las variables ideas y actitudes

\begin{tabular}{|c|c|}
\hline ITEMS IDEAS & ITEMS ACTITUDES \\
\hline $\begin{array}{c}1,3,4,7,8,10,11,13,15, \\
16,17,18,19,20,21,22, \\
23,24,25,26,27,28,29,\end{array}$ & \\
$30,31,32,33$ & $2,5,6,9,12,14$ \\
\hline
\end{tabular}

La decisión de agregar un nuevo ítem responde a las dudas formuladas por los expertos que participaron en el estudio piloto en relación al ítem 3, a los estudiantes con discapacidad les cuesta más terminar una carrera, pues proponen que puede costarles más, pero terminar la carrera en el plazo establecido (cuatro años, por ejemplo, en el caso de los grados), o necesitar más años que sus compañeros sin discapacidad para finalizar sus estudios. 
La incorporación de una nueva alternativa de respuesta se realizó con el fin de evitar un sesgo a favor de un resultado positivo, ya que al añadir ese punto medio se ofrece a los encuestados la posibilidad de seleccionar esta opción en caso de indecisión, evitando así la elección forzada de una opción positiva por la necesidad de ofrecer una imagen de sí más favorable de lo normal y/o quedar bien con el experimentador (deseabilidad social).

De modo general, las categorías en las respuestas a los ítems del cuestionario se establecieron a partir de la pregunta inicial "Con respecto a los estudiantes de la Universidad de Santiago de Compostela que presentan algún tipo de discapacidad, pienso que" (tache con una X la puntuación más adecuada en función del grado de acuerdo-desacuerdo): 1. Totalmente en desacuerdo; 2. En desacuerdo; 3. Indiferente; 4. De acuerdo; 5. Totalmente de acuerdo.

\subsection{Procedimiento}

El procedimiento de recogida de datos se efectuó siguiendo un protocolo homogéneo: todos los cuestionarios fueron administrados por la misma persona, permitiendo de este modo que todos los participantes reciban las mismas explicaciones sobre los objetivos del estudio, y en todos los casos se utilizó el formato papel. La administración se realizó durante varios días, en los que la investigadora se desplazó a las diferentes facultades de la USC en las que se recogieron los datos. Para llevar a cabo el estudio se contó con la colaboración de los Decanatos, que facilitaron el contacto entre el profesorado y la investigadora. Esto permitió llevar a cabo la recogida de información de forma colectiva durante las horas de clase ya que, según Arias y Fernández (1998), este tipo de administración facilita altas tasas de cooperación y mayor anonimato en las respuestas. En todos los casos el profesorado colaborador cedió los 20 primeros minutos de sus clases, durante los cuales se realizó la presentación de la investigadora, que explicó los objetivos del estudio, solicitó la colaboración de los estudiantes y entregó los cuestionarios a aquellos que se mostraron dispuestos a colaborar. Con el fin de garantizar la confidencialidad de las respuestas del alumnado la participación fue anónima y totalmente voluntaria, destacando que la acogida fue muy buena en todos los casos y que ningún estudiante rechazó colaborar en la investigación. El tiempo destinado a leer y cubrir los cuestionarios, unos 15 minutos aproximadamente, fue suficiente en todos los casos. No hubo ninguna consulta en relación a los ítems formulados, probablemente porque todas las afirmaciones eran claras y concisas, ni ninguna incidencia y/o dificultad en el proceso de recogida de información. Dado que los cuestionarios iban dirigidos a población sin discapacidad, no fue necesario realizar adaptaciones de los mismos, si bien se tuvieron en cuenta una serie de aspectos formales para facilitar su lectura: fuente de letra Times New Roman, tamaño de fuente 13 puntos., interlineado 1.5 o repetir el encabezado de las tablas cada vez que se cambiaba de página, para que el alumnado no tuviese que volver a la página anterior a consultar las diferentes opciones de respuesta, entre otros. 


\subsection{Análisis de los datos}

Con el fin de obtener la distribución de las respuestas a cada ítem del cuestionario, así como las medias y las desviaciones de cada uno de los ítems y dimensiones, se han Ilevado a cabo análisis descriptivos. Además, para valorar la incidencia de las variables independientes se ha utilizado el estadístico T-Student (dos muestras independientes) y la prueba ANOVA (variables con más de dos categorías). Todos los análisis estadísticos se han realizado con el paquete estadístico SPSS, en su versión 22 para Windows (IBM, Inc.).

\section{Resultados}

Para facilitar la comprensión de los resultados obtenidos, estos se han agrupado en seis apartados, en base a los objetivos formulados en la investigación: ideas y actitudes de los estudiantes de la USC hacia sus compañeros con diversidad funcional, ideas y actitudes en función de la edad, ideas y actitudes en función del género, ideas y actitudes en función del área de conocimiento, ideas y actitudes en función del curso en el que se encuentran matriculados los estudiantes, e ideas y actitudes en relación a si han recibido o no algún tipo de información en la USC acerca de la presencia de compañeros/as con discapacidad.

Al hablar de actitudes se hace referencia a "aquellas conductas concretas, a desarrollar en pro de la integración educativa y social de estudiantes con discapacidad en la universidad que, por su propia naturaleza, indican un compromiso que va más allá de las ideas" (Sánchez-Palomino, 2009, p. 117).

Para los primeros análisis se han establecido dos categorías de respuesta: en desacuerdo (columnas uno y dos) y de acuerdo (columnas cuatro y cinco), si bien debe tenerse en cuenta, de cara a la interpretación de los datos, que en varias de las preguntas planteadas existen altos porcentajes de indecisión (columna 3).

\subsection{Ideas y actitudes de los estudiantes de la USC hacia sus compañeros con diversidad funcional}

En general, las ideas que tienen los estudiantes de la USC acerca de las capacidades de sus compañeros universitarios con diversidad funcional son positivas (ver Tabla 4), si bien Ilama la atención que en varias de las preguntas planteadas existen altos porcentajes de indecisión lo que, probablemente, responda al desconocimiento de las necesidades y dificultades que tienen sus compañeros y compañeras y/o a la falta de experiencia personal con la discapacidad. 
Tabla 4. Porcentajes de respuesta, medias y desviaciones típicas del factor ideas

\begin{tabular}{|c|c|c|c|c|c|c|c|}
\hline ÍTEMS: Factor Teórico Ideas & 1 & 2 & 3 & 4 & 5 & Media & D.T. \\
\hline $\begin{array}{l}\text { 1. Se debe facilitar el acceso de los estu- } \\
\text { diantes con discapacidad a la Universidad }\end{array}$ & 0.5 & 1.0 & 0.8 & 9.8 & 88.0 & 4.84 & .52 \\
\hline $\begin{array}{l}\text { 3. A los estudiantes con discapacidad les re- } \\
\text { sulta más difícil terminar una carrera }\end{array}$ & 14.5 & 36.1 & 18.8 & 25.6 & 5.0 & 2.70 & 1.15 \\
\hline $\begin{array}{l}\text { 4. Los estudiantes con discapacidad necesi- } \\
\text { tan más años para terminar una carrera }\end{array}$ & 24.0 & 44.0 & 21.3 & 9.3 & 1.5 & 2.20 & .96 \\
\hline $\begin{array}{l}\text { 7. La existencia en las aulas de estudiantes } \\
\text { con discapacidad dificulta el aprendizaje }\end{array}$ & 73.1 & 21.6 & 2.8 & 1.3 & 0.3 & 1.31 & .61 \\
\hline $\begin{array}{l}\text { 8. Los objetivos y contenidos deben ser los } \\
\text { mismos para todos los estudiantes }\end{array}$ & 3.3 & 8.3 & 8.8 & 35.9 & 43.7 & 4.08 & 1.07 \\
\hline $\begin{array}{l}\text { 10. Se deben fomentar actitudes favorables } \\
\text { de cara a la integración de estudiantes con } \\
\text { discapacidad }\end{array}$ & 0.3 & 0.8 & 1.5 & 26.8 & 70.7 & 4.67 & .57 \\
\hline $\begin{array}{l}\text { 11. Se debe facilitar a estos estudiantes el } \\
\text { acceso a los contenidos y a la evaluación } \\
\text { según sus necesidades }\end{array}$ & 0.8 & 5.5 & 4.5 & 35.8 & 53.4 & 4.35 & .86 \\
\hline $\begin{array}{l}\text { 13. Debe existir una unidad central que } \\
\text { coordine y asesore a estudiantes con dis- } \\
\text { capacidad, al profesorado implicado en su } \\
\text { formación y al personal de administración y } \\
\text { servicios. }\end{array}$ & 0.8 & 1.5 & 7.5 & 43.5 & 46.7 & 4.34 & .75 \\
\hline $\begin{array}{l}\text { 15. Debe informarse a los estudiantes de la } \\
\text { existencia en sus aulas de compañeros/as } \\
\text { con discapacidad }\end{array}$ & 12.8 & 18.3 & 24.4 & 30.9 & 13.6 & 3.14 & 1.24 \\
\hline $\begin{array}{l}\text { 16. Debe existir en la Universidad profeso- } \\
\text { rado especializado para estos estudiantes }\end{array}$ & 3.3 & 12.3 & 20.1 & 45.1 & 19.3 & 3.65 & 1.03 \\
\hline $\begin{array}{l}\text { 17. La Universidad debe poner los medios } \\
\text { para que los estudiantes con discapacidad } \\
\text { participen en la vida universitaria como el } \\
\text { resto de sus compañeros/as }\end{array}$ & 0.3 & 1.0 & 1.3 & 28.8 & 68.8 & 4.65 & .59 \\
\hline $\begin{array}{l}\text { 18. En la Universidad deben existir grupos } \\
\text { específicos de clase/aula para los estudian- } \\
\text { tes con discapacidad }\end{array}$ & 23.5 & 43.3 & 16.8 & 14.0 & 2.5 & 2.29 & 1.05 \\
\hline $\begin{array}{l}\text { 19. Es necesario que la Universidad firme } \\
\text { convenios de colaboración con asociacio- } \\
\text { nes e instituciones públicas y/o privadas } \\
\text { para mejorar la atención a los estudiantes } \\
\text { con discapacidad }\end{array}$ & 0.8 & 2.8 & 12.5 & 52.6 & 31.3 & 4.11 & .78 \\
\hline $\begin{array}{l}\text { 20. La Universidad no es el lugar más ade- } \\
\text { cuado para la formación académica y pro- } \\
\text { fesional de los estudiantes con discapacidad }\end{array}$ & 71.9 & 23.1 & 3.3 & 1.0 & 0.8 & 1.35 & .66 \\
\hline
\end{tabular}




\begin{tabular}{|c|c|c|c|c|c|c|c|}
\hline ÍTEMS: Factor Teórico Ideas & 1 & 2 & 3 & 4 & 5 & Media & D.T. \\
\hline $\begin{array}{l}\text { 21. Me supone un problema o dificultad la } \\
\text { presencia en las aulas de estudiantes con } \\
\text { discapacidad }\end{array}$ & 78.9 & 17.3 & 3.0 & 0.5 & 0.3 & 1.26 & .56 \\
\hline $\begin{array}{l}22 \text { Debe existir una normativa específica } \\
\text { que regule la atención a los estudiantes con } \\
\text { discapacidad en la Universidad }\end{array}$ & 3.8 & 9.8 & 27.9 & 47.0 & 11.6 & 3.53 & .95 \\
\hline $\begin{array}{l}\text { 23. La Universidad dispone de los medios para } \\
\text { solucionar cualquier tipo de adaptación en el } \\
\text { acceso al currículum de estos estudiantes }\end{array}$ & 11.6 & 28.7 & 38.4 & 15.5 & 5.8 & 2.75 & 1.04 \\
\hline $\begin{array}{l}\text { 24. Se debe tener con ellos más flexibilidad } \\
\text { a la hora de exigirles la adquisición de las } \\
\text { competencias propias de las titulaciones/ } \\
\text { carreras }\end{array}$ & 15.4 & 34.6 & 22.2 & 24.7 & 3.0 & 2.65 & 1.10 \\
\hline $\begin{array}{l}\text { 25. La presencia en las aulas de estudiantes } \\
\text { con discapacidad beneficia a todos/as }\end{array}$ & 4.0 & 6.8 & 38.8 & 31.6 & 18.8 & 3.54 & 1.00 \\
\hline $\begin{array}{l}\text { 26. Se deben incrementar los esfuerzos eco- } \\
\text { nómicos para la atención a los estudiantes } \\
\text { con discapacidad }\end{array}$ & 1.3 & 3.8 & 13.3 & 53.1 & 28.6 & 4.04 & .82 \\
\hline $\begin{array}{l}\text { 27. La presencia en las aulas de estudiantes } \\
\text { con discapacidad produce un descenso ge- } \\
\text { neralizado del nivel académico }\end{array}$ & 65.1 & 26.9 & 5.5 & 1.0 & 1.5 & 1.47 & .78 \\
\hline $\begin{array}{l}\text { 28. Los estudiantes con discapacidad deben } \\
\text { tener las mismas oportunidades y posibilidades } \\
\text { de promoción que los demás universitarios/as }\end{array}$ & 0.3 & 0.8 & 2.0 & 21.8 & 75.3 & 4.71 & .57 \\
\hline $\begin{array}{l}\text { 29. Las pruebas de acceso a la universidad } \\
\text { deben contemplar los recursos y adaptacio- } \\
\text { nes necesarias para dar respuesta a las nece- } \\
\text { sidades de los estudiantes con discapacidad }\end{array}$ & 0.8 & 4.5 & 9.0 & 46.5 & 39.2 & 4.19 & .83 \\
\hline $\begin{array}{l}\text { 30. La titulación alcanzada por los estu- } \\
\text { diantes con discapacidad debe ser garantía } \\
\text { del conjunto de competencias para su ejer- } \\
\text { cicio profesional }\end{array}$ & 0.3 & 1.8 & 10.8 & 42.6 & 44.6 & 4.29 & .75 \\
\hline $\begin{array}{l}\text { 31. Los estudiantes, especialmente aquellos } \\
\text { que presentan alguna discapacidad, deben } \\
\text { conocer las competencias que se exigirán } \\
\text { en cada titulación/carrera antes de formali- } \\
\text { zar su matrícula. }\end{array}$ & 1.5 & 3.5 & 12.5 & 46.9 & 35.6 & 4.11 & .86 \\
\hline $\begin{array}{l}\text { 32. Es necesario que la Universidad desa- } \\
\text { rrolle un Plan de Acogida para los estudian- } \\
\text { tes con discapacidad }\end{array}$ & 1.0 & 4.3 & 17.8 & 54.6 & 22.3 & 3.92 & .81 \\
\hline $\begin{array}{l}\text { 33. Es necesario que exista comunicación } \\
\text { entre compañeros/as como apoyo y ayuda a } \\
\text { estudiantes con discapacidad }\end{array}$ & 0.2 & 2.3 & 7.8 & 44.0 & 45.7 & 4.33 & .74 \\
\hline
\end{tabular}


Cabe destacar que el 95\% de los encuestados considera que la Universidad es el lugar adecuado para la formación académica y profesional de estudiantes con discapacidad y que el $85.7 \%$ indica que las pruebas de acceso a la universidad deben contemplar los recursos y adaptaciones necesarias para dar respuesta a las necesidades de estos estudiantes. Si se tiene en cuenta el alto consenso expresado y el bajo porcentaje de respuestas neutras en ambos casos (ítem 20 e ítem 29, respectivamente), se puede afirmar que los estudiantes de la USC confían en las capacidades de sus compañeros con discapacidad para llevar los estudios universitarios adelante. Esta idea se confirma con el hecho de que el 66.8\% apoyan que no deben existir grupos específicos de clase/aula para los estudiantes con discapacidad (ítem 18), lo que indicaría, por tanto, que los estudiantes de la USC se muestran partidarios de la integración educativa a nivel universitario.

No obstante, existe cierta controversia en cuanto a las capacidades de sus compañeros con discapacidad, pues los datos indican que existen dudas acerca de si a los estudiantes con discapacidad les cuesta más, o no, terminar una carrera (ítem 3) y, por tanto, a si necesitan más años, o no, para terminarla (ítem 4). Estas dudas se traducen en que para el $50 \%$ no se debe tener con ellos más flexibilidad a la hora de exigirles la adquisición de las competencias propias de las titulaciones/carrera, mientras que el $27.3 \%$ considera que sí, existiendo un alto porcentaje de indecisos al respecto (ítem 24).

En cuanto a las ideas sobre la formación de los iguales con discapacidad, existe un alto consenso en relación a que se facilite el acceso a la universidad de los estudiantes con discapacidad (ítem 1), a que la Universidad ponga los medios para que la integración sea posible (ítem 17) y a que se facilite a los estudiantes con discapacidad el acceso a los contenidos y a la evaluación, según sus necesidades (ítem 11). Además, un alto porcentaje de encuestados (90.2\%) piensa que debe existir una unidad central que coordine y asesore a estudiantes con discapacidad, al profesorado y al personal de administración y servicios (ítem 13).

La gran mayoría de los encuestados están de acuerdo en que la presencia de estudiantes con discapacidad en las aulas universitarias no produce un descenso generalizado del nivel académico (92\%, ítem 27), no les supone un problema o dificultad (96,2\%, ítem 21), ni dificulta el aprendizaje (ítem 7).

Existen dudas respecto a si deben ser informados sobre la presencia de compañeros con discapacidad (ítem 15), a si existen medios en la Universidad para realizar adaptaciones de acceso al currículum (ítem 23) y a si se necesita profesorado especializado para atender al alumnado con discapacidad (ítem 16).

Se deduce, de lo expuesto anteriormente, que los estudiantes de la USC responsabilizan a la propia institución académica de que los compañeros con discapacidad reciban, o no, una adecuada formación.

En relación al desarrollo profesional, destaca una vez más la buena disposición de cara a la integración (ítem 33) y la defensa aplastante del principio de igualdad de oportunidades, pues el $\mathbf{9 7 . 1 \%}$ piensa que los estudiantes con discapacidad deben tener las mismas oportunidades y posibilidades de promoción que los demás universitarios/as (ítem 28), si bien se hace constar que esta integración no debe compro- 
meter la formación recibida al indicar, el $86.8 \%$, que la titulación alcanzada por los estudiantes con discapacidad debe ser garantía del conjunto de competencias para su ejercicio profesional (ítem 30).

Hay un 38.8\% de los encuestados que muestran indiferencia ante la afirmación de que la presencia de alumnado con discapacidad en el aula beneficia a todos (ítem 25). Quizás, este dato podría ser el reflejo de una normalización educativa en la universidad, al reflejar que todos somos iguales y, por tanto, la presencia de estudiantes con discapacidad no tendría por qué beneficiar (ni por qué perjudicar) a nadie.

Como puede observarse a continuación (ver Tabla 5), al igual que ocurría en el caso de las ideas, las actitudes de los estudiantes de la USC hacia sus compañeros con discapacidad son positivas, destacando que no les causa problema tener en clase compañeros con discapacidad (ítem 2) y que, mayoritariamente ( $85.7 \%)$, se sienten preparados/as para relacionarse con ellos, sin que dicho contacto les produzca ansiedad ni dificultad alguna.

Otros datos positivos de cara a la inclusión de los estudiantes con discapacidad en la Universidad de Santiago de Compostela lo constituyen el hecho de que el $72.9 \%$ de sus compañeros sin discapacidad están dispuestos a informarse para facilitar su estancia en la USC y que, además, el $63.7 \%$ tiene un trato hacia ellos de igual a igual, sin sentimientos de pena, compasión o lástima por la condición de persona con discapacidad (ítem 9). No obstante, respecto a este último ítem, cabe destacar que un $18.2 \%$ sí ha sentido pena, compasión o lástima en alguna ocasión o no ha valorado sus capacidades y que el mismo porcentaje de encuestados (18.2\%) se ha mostrado indiferente ante este ítem, algo que pudiera ser debido a la falta de experiencia personal con la discapacidad y/o a las dificultades para contestar a esta pregunta, pues una respuesta positiva implicaría ofrecer una imagen de sí mismo más desfavorable de lo esperado.

Tabla 5. Porcentajes de respuesta, medias y desviaciones típicas del factor actitudes

\begin{tabular}{|l|c|c|c|c|c|c|c|}
\hline \multicolumn{1}{|c|}{ ÍTEMS: Factor Teórico Actitudes } & $\mathbf{1}$ & $\mathbf{2}$ & $\mathbf{3}$ & $\mathbf{4}$ & $\mathbf{5}$ & Media & D.T. \\
\hline $\begin{array}{l}\text { 2. Prefiero no tener compañeros y compa- } \\
\text { ñeras con discapacidad en clase }\end{array}$ & 84.8 & 6.5 & 0.0 & 8.3 & 0.5 & $\mathbf{1 . 2 5}$ & $\mathbf{. 6 2}$ \\
\hline $\begin{array}{l}\text { 5. Me produce ansiedad trabajar con com- } \\
\text { pañeros y compañeras con discapacidad }\end{array}$ & 71.9 & 17.6 & 8.0 & 1.5 & 1.0 & $\mathbf{1 . 4 2}$ & $\mathbf{. 7 9}$ \\
\hline $\begin{array}{l}\text { 6. Tengo más dificultad para relacionarme } \\
\text { con compañeros/as que presentan alguna } \\
\text { discapacidad }\end{array}$ & 50.3 & 28.1 & 12.8 & 8.3 & 0.5 & $\mathbf{1 . 8 1}$ & $\mathbf{. 9 9}$ \\
\hline $\begin{array}{l}\text { 9. Siento pena, compasión o lástima por los } \\
\text { estudiantes con discapacidad y tiendo a ayu- } \\
\text { darles en exceso, sin valorar sus capacidades }\end{array}$ & 26.3 & 37.4 & 18.2 & 17.7 & 0.5 & $\mathbf{2 . 2 9}$ & $\mathbf{1 . 0 6}$ \\
\hline $\begin{array}{l}\text { 12. Estoy dispuesto/a a informarme para fa- } \\
\text { cilitar su estancia en la Universidad }\end{array}$ & 0.8 & 7.6 & 18.1 & 50.6 & 22.9 & $\mathbf{3 . 8 7}$ & $\mathbf{. 8 8}$ \\
\hline $\begin{array}{l}\text { 14. Me siento preparado/a para relacionar- } \\
\text { me con estudiantes con discapacidad en la } \\
\text { Universidad }\end{array}$ & 1.0 & 6.0 & 7.3 & 42.0 & 43.7 & $\mathbf{4 . 2 1}$ & $\mathbf{. 8 9}$ \\
\hline
\end{tabular}




\subsection{Ideas y actitudes en relación a la variable edad}

Para poder llevar a cabo los análisis en relación a esta variable, se ha agrupado a los estudiantes en cuatro intervalos de edad y se ha calculado la media y la desviación típica de ambos factores (ideas y actitudes) (ver Tabla 6).

Tabla 6. Frecuencia-porcentajes de la variable edad (intervalos) y medias-desviaciones típicas de los factores teóricos analizados

\begin{tabular}{|c|c|c|c|c|c|c|}
\hline $\begin{array}{l}\text { INTERVALO } \\
\text { DE EDAD }\end{array}$ & FRECUENCIA & PORCENTAJE & \multicolumn{2}{|c|}{$\begin{array}{c}\text { FACTOR TEÓRICO } \\
\text { IDEAS }\end{array}$} & \multicolumn{2}{|c|}{$\begin{array}{c}\text { FACTOR TEÓRICO } \\
\text { ACTITUDES }\end{array}$} \\
\hline$\leq 18$ & 36 & 9.00 & $\mathbf{x}$ & $\sigma$ & $\mathbf{x}$ & $\sigma$ \\
\hline $19-28$ & 341 & 85.25 & \multirow{4}{*}{3.41} & \multirow{4}{*}{.53} & \multirow{4}{*}{2.32} & \multirow{4}{*}{.51} \\
\hline $29-38$ & 20 & 5.00 & & & & \\
\hline $39-45$ & 3 & 0.75 & & & & \\
\hline TOTAL & 400 & 100.00 & & & & \\
\hline
\end{tabular}

Teniendo en cuenta la media y la desviación típica de los factores teóricos (ver Tabla 6), para analizar si la variable edad influye en las ideas y/o actitudes que los estudiantes de la USC tienen hacia sus compañeros con diversidad funcional, se hace necesario llevar a cabo un análisis sobre el modelo de comparación de medias, por lo que se utiliza un análisis lineal univariante con análisis de varianza factorial, donde la variable dependiente es la edad y las independientes los factores teóricos ideas y actitudes (ver Tabla 7).

Tabla 7. Análisis de varianza factorial

\begin{tabular}{|l|c|c|c|c|c|}
\hline \multicolumn{1}{|c|}{ Fuente } & $\begin{array}{c}\text { Suma de } \\
\text { cuadrados } \\
\text { tipo III }\end{array}$ & g.I. & $\begin{array}{c}\text { Media } \\
\text { cuadrática }\end{array}$ & F & $\begin{array}{c}\text { Significación } \\
\text { (p) }\end{array}$ \\
\hline Modelo corregido & $1550.686^{(a)}$ & 11 & 140.971 & 904.528 & .000 \\
\hline IDEAS & .343 & 2 & .171 & 1.100 & .334 \\
\hline ACTITUDES & .575 & 3 & 192 & 1.229 & .299 \\
\hline IDEAS x ACTITUDES & 1.312 & 5 & .262 & 1.684 & .137 \\
\hline
\end{tabular}

(a) $\mathrm{R}$ cuadrado $=, 963$ ( $\mathrm{R}$ cuadrado corregida $=, 961$ )

Tomando como criterio que el valor de p (significación) sea menor de .05 (nivel de confianza de 95\%) para decidir la existencia de diferencias significativas se observa, en la fila modelo corregido de la tabla anterior (ver Tabla 7), que el estadístico F presenta un nivel crítico $p=.000$, lo que indica que el modelo es fiable para la variable edad. En las dos filas siguientes, ideas y actitudes, se recogen los efectos de las variables independientes. Si se tienen en cuenta los niveles críticos de ambas (.334 y .299, respectivamente), puede concluirse que no hay diferencias significativas para los distintos grupos de edad. La interacción ideas y actitudes respecto a la variable 
dependiente edad aparece en la última fila. Teniendo en cuenta los datos que en ella aparecen $(p=.137)$, se deduce que no existen diferencias significativas entre ellas.

A la vista de lo expuesto anteriormente, puede deducirse que, con independencia de su edad, los estudiantes de la USC manifiestan unas ideas positivas, de aceptación (de acuerdo o totalmente de acuerdo), hacia la integración de estudiantes con necesidades especiales asociadas a una discapacidad en la universidad.

\subsection{Ideas y actitudes en relación al género}

Para el análisis de la relación entre ideas y género, así como entre actitudes y género, se utilizó el análisis de contingencias, tomando como variable independiente el género. Los resultados obtenidos indican que no existen diferencias estadísticamente significativas, en relación al género, respecto a la integración del alumnado con discapacidad en la universidad (ver Tabla 8).

Tabla 8. Ideas/Actitudes-Género

\begin{tabular}{|c|c|c|c|c|c|c|c|}
\hline & \multicolumn{5}{|c|}{ Factor Teórico Ideas } & \multirow[b]{2}{*}{ Total } \\
\hline & & $\begin{array}{c}\text { Totalmente } \\
\text { en } \\
\text { desacuerdo }\end{array}$ & $\begin{array}{c}\text { En } \\
\text { desacuerdo }\end{array}$ & Indiferente & $\begin{array}{c}\text { De } \\
\text { acuerdo }\end{array}$ & $\begin{array}{l}\text { Totalmente } \\
\text { de acuerdo }\end{array}$ & \\
\hline \multirow{2}{*}{ Género } & Hombre & 0 & 5 & 76 & 41 & 0 & 122 \\
\hline & Mujer & 0 & 3 & 143 & 132 & 0 & 278 \\
\hline \multicolumn{2}{|l|}{ Total } & $\mathbf{0}$ & 8 & 219 & 173 & $\mathbf{0}$ & 400 \\
\hline & & \multicolumn{5}{|c|}{ Factor Teórico Actitudes } & \\
\hline & & $\begin{array}{c}\text { Totalmente } \\
\text { en } \\
\text { desacuerdo }\end{array}$ & $\begin{array}{c}\text { En } \\
\text { desacuerdo }\end{array}$ & Indiferente & $\begin{array}{c}\text { De } \\
\text { acuerdo }\end{array}$ & $\begin{array}{l}\text { Totalmente } \\
\text { de acuerdo }\end{array}$ & Total \\
\hline \multirow{2}{*}{ Género } & Hombre & 1 & 81 & 39 & 1 & 0 & 122 \\
\hline & Mujer & 5 & 180 & 91 & 2 & 0 & 278 \\
\hline \multicolumn{2}{|l|}{ Total } & 6 & 261 & 130 & 3 & $\mathbf{0}$ & 400 \\
\hline & & \multicolumn{3}{|c|}{ IDEAS } & \multicolumn{3}{|c|}{ ACTITUDES } \\
\hline & & $\mathbf{x}$ & \multicolumn{2}{|c|}{$\sigma$} & $x$ & \multicolumn{2}{|l|}{$\sigma$} \\
\hline \multirow{2}{*}{ Género } & Hombre & \multicolumn{2}{|l|}{3.29} & .54 & 2.33 & \multicolumn{2}{|l|}{.51} \\
\hline & Mujer & 3.46 & \multicolumn{2}{|c|}{.52} & 2.32 & \multicolumn{2}{|l|}{.52} \\
\hline
\end{tabular}

\subsection{Ideas y actitudes en relación a la variable área de conocimiento}

En relación al área de conocimiento en la que el alumnado cursa sus estudios, puede verse la distribución de la muestra en el apartado de método (ver Tabla 1). 
Al igual que en el caso del género, para el análisis comparativo y la valoración de los factores teóricos ideas y actitudes por área de conocimiento se utilizó el análisis de contingencias observando que, en general, en todos los casos hay una buena aceptación de la integración del alumnado con discapacidad en la universidad, si bien se observa un nivel comparativo más alto ( $p \leq .001)$, en relación a las ideas, en las áreas de Ciencias de la Salud, Ciencias Jurídicas y Sociales y Humanidades con respecto a las áreas de Ciencias Experimentales y de Enseñanzas Técnicas. En cuanto a las actitudes, no se observan diferencias estadísticamente significativas (ver Tabla 9).

Tabla 9. Ideas/Actitudes-Área de conocimiento

\begin{tabular}{|c|c|c|c|c|c|c|}
\hline & \multicolumn{5}{|c|}{ Factor Teórico Ideas } & \multirow[b]{2}{*}{ Total } \\
\hline & $\begin{array}{c}\text { Totalmente } \\
\text { en } \\
\text { desacuerdo }\end{array}$ & $\begin{array}{c}\text { En } \\
\text { desacuerdo }\end{array}$ & Indiferente & $\begin{array}{c}\text { De } \\
\text { acuerdo }\end{array}$ & $\begin{array}{l}\text { Totalmente } \\
\text { de acuerdo }\end{array}$ & \\
\hline $\begin{array}{l}\text { Ciencias de la } \\
\text { salud }\end{array}$ & 0 & 0 & 46 & 32 & 0 & 78 \\
\hline $\begin{array}{l}\text { Ciencias } \\
\text { Experimentales }\end{array}$ & 0 & 3 & 27 & 11 & 0 & 41 \\
\hline $\begin{array}{l}\text { Ciencias } \\
\text { jurídicas y } \\
\text { sociales }\end{array}$ & 0 & 3 & 99 & 103 & 0 & 205 \\
\hline $\begin{array}{l}\text { Enseñanzas } \\
\text { técnicas }\end{array}$ & 0 & 2 & 23 & 11 & 0 & 36 \\
\hline Humanidades & 0 & 0 & 24 & 16 & 0 & 40 \\
\hline TOTAL & $\mathbf{0}$ & 8 & 219 & 173 & $\mathbf{0}$ & 400 \\
\hline
\end{tabular}

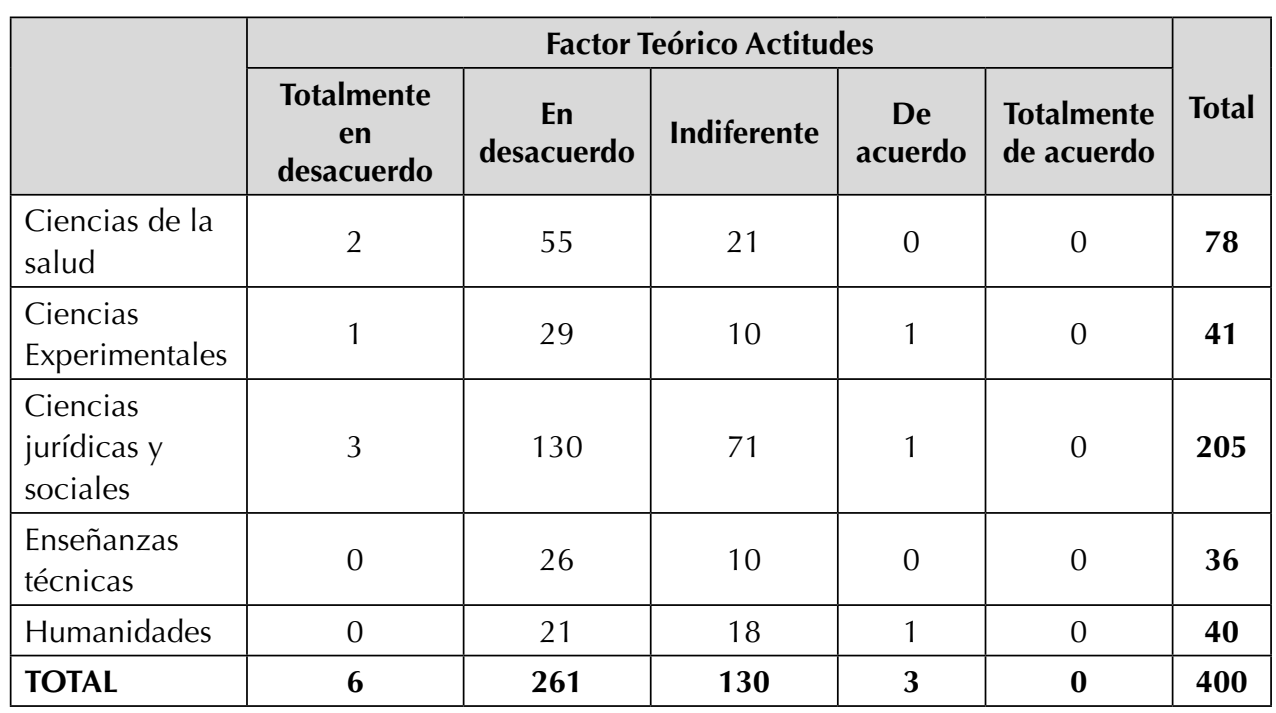




\begin{tabular}{|l|c|c|c|c|}
\hline \multirow{2}{*}{} & \multicolumn{2}{|c|}{ IDEAS } & \multicolumn{2}{c|}{ ACTITUDES } \\
\cline { 2 - 5 } & $\mathrm{x}$ & $\sigma$ & $\mathbf{x}$ & $\sigma$ \\
\hline $\begin{array}{l}\text { Ciencias de la } \\
\text { salud }\end{array}$ & 3.41 & .50 & $\mathbf{2 . 2 4}$ & .59 \\
\hline $\begin{array}{l}\text { Ciencias } \\
\text { Experimentales }\end{array}$ & $\mathbf{3 . 1 9}$ & .56 & 2.27 & .55 \\
\hline $\begin{array}{l}\text { Ciencias } \\
\text { jurídicas y } \\
\text { sociales }\end{array}$ & 3.49 & .53 & 2.34 & .51 \\
\hline $\begin{array}{l}\text { Enseñanzas } \\
\text { técnicas }\end{array}$ & 3.25 & .55 & $\mathbf{2 . 2 7}$ & .45 \\
\hline Humanidades & $\mathbf{3 . 4 0}$ & .49 & $\mathbf{2 . 5 0}$ & .55 \\
\hline
\end{tabular}

\subsection{Ideas y actitudes en relación a la variable curso académico}

Del análisis de las medias se desprende que no hay diferencias significativas entre las actitudes e ideas que presentan los estudiantes de los diferentes cursos (ver Tabla 10). Estos datos son coherentes con los presentados en relación a la variable edad (ver Tabla 7), puesto que a mayor curso académico, mayor es la edad del alumnado.

Tabla 10. Ideas/Actitudes-Curso académico

\begin{tabular}{|l|c|c|c|c|c|c|}
\hline & \multicolumn{5}{|c|}{ Factor Teórico Ideas } & \multirow{2}{*}{ Total } \\
\cline { 2 - 7 } & $\begin{array}{c}\text { Totalmente en } \\
\text { desacuerdo }\end{array}$ & $\begin{array}{c}\text { En } \\
\text { desacuerdo }\end{array}$ & Indiferente & $\begin{array}{c}\text { De } \\
\text { acuerdo }\end{array}$ & $\begin{array}{c}\text { Totalmente } \\
\text { de acuerdo }\end{array}$ & \\
\hline PRIMERO & 0 & 3 & 44 & 45 & 0 & $\mathbf{9 2}$ \\
\hline SEGUNDO & 0 & 1 & 57 & 33 & 0 & $\mathbf{9 1}$ \\
\hline TERCERO & 0 & 1 & 34 & 14 & 0 & $\mathbf{4 9}$ \\
\hline CUARTO & 0 & 3 & 55 & 49 & 0 & $\mathbf{1 0 7}$ \\
\hline QUINTO & 0 & 0 & 22 & 24 & 0 & $\mathbf{4 6}$ \\
\hline SEXTO & 0 & 0 & 7 & 8 & 0 & $\mathbf{1 5}$ \\
\hline TOTAL & $\mathbf{0}$ & $\mathbf{8}$ & $\mathbf{2 1 9}$ & $\mathbf{1 7 3}$ & $\mathbf{0}$ & $\mathbf{4 0 0}$ \\
\hline
\end{tabular}

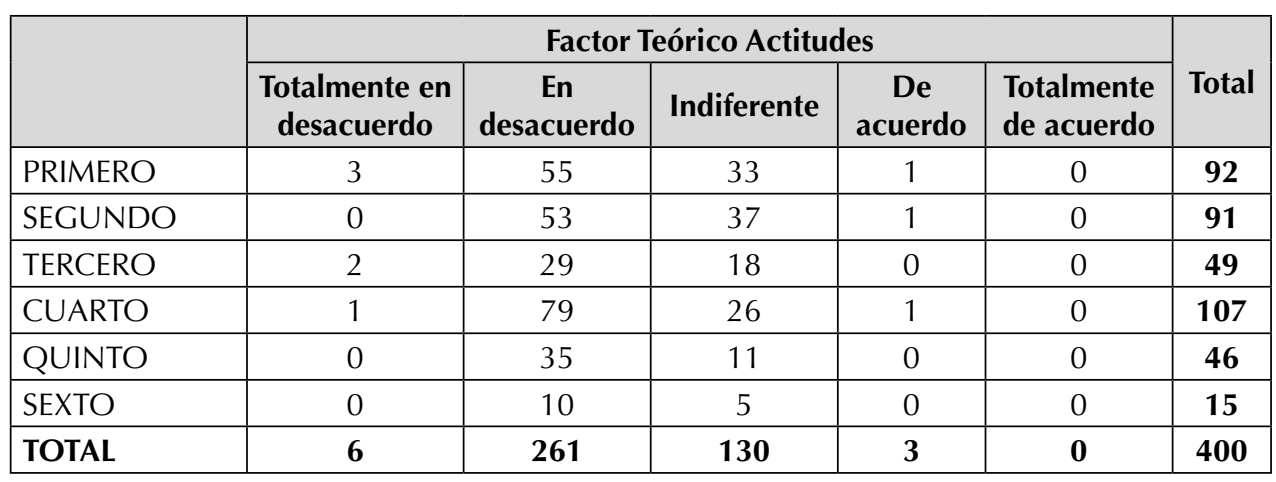




\begin{tabular}{|l|c|c|c|c|}
\hline \multirow{2}{*}{} & \multicolumn{2}{|c|}{ IDEAS } & \multicolumn{2}{c|}{ ACTITUDES } \\
\cline { 2 - 5 } & $\mathbf{x}$ & $\sigma$ & $\mathbf{x}$ & $\sigma$ \\
\hline PRIMERO & 3.46 & .56 & 2.35 & .56 \\
\hline SEGUNDO & 3.35 & .50 & 2.43 & .52 \\
\hline TERCERO & 3.26 & .49 & 2.33 & .55 \\
\hline CUARTO & 3.43 & .55 & 2.25 & .48 \\
\hline QUINTO & 3.52 & .51 & 2.24 & .43 \\
\hline SEXTO & 3.53 & .52 & 2.33 & .48 \\
\hline
\end{tabular}

\subsection{Ideas y actitudes en relación a la variable "he recibido algún tipo de información en la USC acerca de la presencia de compañeros/as con discapacidad"}

En este apartado cabe destacar que sólo el 1.5\% de los encuestados ha manifestado haber recibido información, en la USC, acerca del colectivo de estudiantes con discapacidad. Las medias, tanto en base a las ideas como a las actitudes, son muy similares entre los que han recibido información y los que no. Este dato indicaría, por tanto, que el haber recibido esta información no parece condicionar las ideas y actitudes de los estudiantes hacia sus compañeros con diversidad funcional (ver Tabla 11).

Tabla 11. Ideas/Actitudes-Información

\begin{tabular}{|c|c|c|}
\hline & \multicolumn{2}{|c|}{ IDEAS } \\
\hline & Media & Desviación Típica \\
\hline Sí han recibido información & 3.50 & .54 \\
\hline \multirow[t]{3}{*}{ No han recibido ningún tipo de información } & 3.41 & .53 \\
\hline & \multicolumn{2}{|c|}{ ACTITUDES } \\
\hline & Media & Desviación Típica \\
\hline Sí han recibido información & 2.33 & .51 \\
\hline No han recibido ningún tipo de información & 2.32 & .51 \\
\hline
\end{tabular}

\section{Discusión y CONCLUSIONES}

Conocer las ideas y actitudes de los estudiantes universitarios sobre sus iguales con diversidad funcional (discapacidad) se hace imprescindible para valorar el nivel de efectividad de las acciones realizadas en niveles inferiores en materia de inclusión educativa y social.

La presente investigación se ha centrado en el caso concreto de la Universidad de Santiago de Compostela (USC), una de las tres universidades públicas de la Comuni- 
dad Autónoma de Galicia (España), donde la matrícula de estudiantes con diversidad funcional ha experimentado un extraordinario aumento en los últimos años. Mientras que en el curso académico 2005/2006 había matriculados un total de 17 estudiantes con discapacidad, en el curso académico 2019/2020 la cifra asciende a más de doscientos.

Tras los análisis realizados estamos en condiciones de afirmar que los estudiantes de la USC manifiestan unas ideas y actitudes positivas, de aceptación hacia la integración de sus compañeros y compañeras con diversidad funcional. Se puede concluir, a partir de los resultados obtenidos, que los estudiantes de la USC son partidarios de facilitar el acceso a la universidad de sus compañeros/as con diversidad funcional, algo que ya se había observado en investigaciones previas realizadas en otras universidades (Luque de la Rosa, 2010; Muñoz-Cantero, Novo-Corti y Espiñeira-Bellón, 2013; Sánchez-Palomino, 2009; Verdugo y Arias, 1991). Además, en concordancia con lo señalado por Valenciano (2009), consideran que la universidad es el lugar más adecuado para la promoción académica y profesional del alumnado con discapacidad, con las mismas oportunidades y posibilidades de promoción que el resto de sus compañeros.

Con independencia de su edad, manifiestan unas ideas positivas, de aceptación hacia la integración en la universidad de estudiantes con necesidades especiales asociadas a una discapacidad. Este resultado difiere de lo encontrado en otros trabajos, en los que los estudiantes más jóvenes muestran una actitud más inclusiva que el resto del alumnado (Martínez, 2010; Moneo y Anaut, 2017 y Sánchez-Palomino, 2009).

Como ocurre en otros trabajos previos (Luque de la Rosa, 2010; Martínez, 2010; Moreno et al., 2006 y Sánchez-Palomino, 2009), no se han encontrado diferencias significativas entre las ideas y actitudes que presentan hombres y mujeres respecto a la integración del alumnado con diversidad funcional en la universidad. No obstante, este resultado no coincide con los de Moneo y Anaut (2017), en cuyo trabajo informan que las mujeres tienen una percepción más positiva hacia las personas con discapacidad que sus pares masculinos.

Se ha encontrado, además, que el curso académico en el que se está matriculado tampoco ofrece diferencias significativas, ni en las ideas ni en las actitudes, con respecto a la integración del alumnado con discapacidad, resultado que es coherente con los obtenidos en relación a la variable edad, puesto que a mayor curso académico, mayor es la edad del alumnado. Con respecto a esta variable cabe destacar que los resultados obtenidos en diferentes investigaciones muestran discrepancias entre ellos, de modo que mientras que Abu-Hamour (2013) detectó actitudes más positivas en el alumnado con menos años de experiencia, Alonso et al. (2008) las hallaron en los estudiantes de último curso.

En relación con las variables mencionadas anteriormente (edad, curso académico y género), los resultados obtenidos se consideran altamente positivos por sus implicaciones pues, aunque sea a modo de hipótesis, apuntan a que la formación, la concienciación y/o la experiencia previa del alumnado en niveles educativos inferiores podría ser la base para facilitar la igualdad de oportunidades, la no discriminación y 
la inclusión social plena de sus pares con diversidad funcional, con quienes compartirán diferentes experiencias de vida tanto en un nivel educativo superior, la universidad, como a nivel laboral, personal y social.

En línea con dicha perspectiva positiva, se observa una buena aceptación de la integración del alumnado con discapacidad en la universidad, si bien existe un nivel comparativo más alto en las áreas de Ciencias de la Salud, Ciencias Jurídicas y Sociales y Humanidades, coincidiendo estos resultados con los de Alonso, Navarro y Lidón (2008), o con otros más recientes (Garabal-Barbeira, Pousada y Espinosa, 2018 y Rodríguez-Martín y Álvarez-Arregui, 2015). Esto puede deberse, como indican los propios autores de estos estudios, a que en las ramas Sociales, Jurídicas y en las de Ciencias de la Salud, se trabaja el conocimiento de la discapacidad como contenido en diversas materias.

Muy pocos alumnos son informados, en la USC, sobre la presencia de compañeros o compañeras con discapacidad, sin que ello influya en sus ideas y actitudes.

Si bien en líneas generales los resultados obtenidos son positivos, no se puede ignorar que un porcentaje nada insignificante, el $18.2 \%$ de los estudiantes encuestados, constata la existencia de ideas y actitudes poco inclusivas, como los sentimientos de pena, compasión o lástima hacia sus iguales con discapacidad y/o la falta de reconocimiento hacia las capacidades de los mismos.

A modo de conclusión final cabe destacar que, si bien el reto de la inclusión sigue presente, tanto dentro como fuera del ámbito educativo universitario, los resultados obtenidos permiten pensar en un futuro prometedor en cuanto a la inclusión socioeducativa.

De cara al futuro, se hace necesario seguir investigando en diferentes universidades, tanto a nivel nacional como internacional, con el fin de encontrar evidencias empíricas que confirmen y proporcionen validez a los resultados obtenidos. También se deberían ampliar los estudios para dar voz al alumnado con diversidad funcional con el propósito de conocer cuáles son las necesidades y las dificultades que presentan.

\section{LimitaCiOnes del eStudio}

A pesar de la importancia de los resultados obtenidos, el tamaño de la muestra impide extrapolarlos al conjunto de los estudiantes universitarios españoles. Pese a esto, debe tenerse en cuenta que los resultados obtenidos en la Universidad de Santiago de Compostela aportan validez a estudios realizados con anterioridad en otras universidades, tanto a nivel nacional como internacional.

Sería interesante, de cara a futuras investigaciones, evaluar el comportamiento de los estudiantes y su evolución en un estudio longitudinal, con el fin de comprobar si existen cambios en sus respuestas y actitudes hacia la diversidad funcional de sus compañeros/as. Además, se podrían incluir otras variables relevantes en la actitud hacia la discapacidad como, por ejemplo, el tipo concreto de discapacidad o el grado en que ésta se manifiesta, ya que podrían influir en las opiniones y conductas (Stovall 
y Sedlacek, 1983). También se podrían considerar otras variables que puedan influir en la percepción de la diversidad como, por ejemplo, la presencia de alguna persona cercana con discapacidad en el ámbito del estudiante universitario.

\section{REFERENCIAS BIBLIOGRÁFICAS}

Abu-Hamour, B. (2013). Faculty Attitudes toward Students with Disabilities in a Public University in Jordan. International Education Studies, 6, 74-81. http://doi. org/10.5539/ies.v6n12p74

Alonso, M., Navarro, R. y Lidón, V. (2008). Actitudes hacia la diversidad en estudiantes universitarios. Fórum de Recerca, 13, 476-491.

Alonso, M. J., Navarro, R. y Vicente, L. (2008). Actitudes hacia la diversidad en estudiantes universitarios. Comunicación presentada en las XIII Jornadas de Fomento de la Investigación. Universitat Jaume I, Castellón, España.

Arias, A. y Fernández, B. (1998). La encuesta como técnica de investigación social. En A. J. Rojas, J. S. Fernández y C. Pérez (Coords.), Investigar mediante encuestas. Fundamentos teóricos y aspectos prácticos (pp. 31-44). Madrid: Síntesis.

Bernal, P. (2018). La investigación en Ciencias Sociales: Técnicas de recolección de la información. Bogotá, Colombia: Universidad Piloto de Colombia.

Bueno, A. (2010). Una mirada ilusionada al futuro de los Servicios de Apoyo a la Discapacidad en a Universidad. Buenas prácticas de apoyo a la discapacidad en la universidad. En A. Bueno (Coord.), Buenas prácticas durante los estudios universitarios en la recepción de la información (pp. 10-33). Alicante: DEE Limencop.

Esteban-Guitart, M., Rivas, M. J. y Pérez, M. R. (2012). Empatía y tolerancia a la diversidad en un contexto educativo intercultural. Universitas Psychologica, 11, 415-426.

Flórez, M. A., Aguado, A. L. y Alcedo, M. A. (2009). Revisión y análisis de los programas de cambio de actitudes hacia personas con discapacidad. Anuario de Psicología Clínica y de la Salud, 5, 85-98.

Fundación ONCE (2014). Formación curricular en Diseño para Todas las Personas (en línea). Recuperado de http://bit.ly/2vlnmoV (Consultado el 02/09/2019).

Fundación Universia (2014). Universidad y discapacidad. Estudio sobre el grado de inclusión del sistema universitario español respecto de la realidad de la discapacidad. Madrid: Fundación Universia y CERMI.

Garabal-Barbeira, J., Pousada, T. y Espinosa, P. C. (2018). Las actitudes como factor clave en la inclusión universitaria. Revista Española de Discapacidad, 6, 181-198. http://doi.org/10.5569/2340-5104.06.01.09

Gughwan, C. y Chow, L. (2001). Korean students' differential attitudes toward people with disabilities: an acculturation perspective. International Journal of Rehabilitation Research, 24, 79-81. http://doi.org/10.1097/00004356-200103000-00012 
Guzmán-Zamora, J. y Sánchez-Palomino, A. (2011). La integración Educativa y Social de Estudiantes con Discapacidad en las Instituciones de Educación Superior del Estado de Tlaxcala (México). Tlaxcala, México: Universidad de Tlaxcala.

Hernández-Garre, C. M., Fernández-Martínez, M. M., Carrión-Martínez, J. J. y AvilésSoler, B. (2019). La inclusión socioeducativa en I Universidad de Minho. Percepciones y actitudes sobre el alumnado con discapacidad. Revista Complutense de Educación, 30, 1097-1112. http://doi.org/10.5209/rced.60106

Huete, A. (2017). Las personas con discapacidad en la escuela. Luces y sombras del proceso de inclusión educativa en España. Panorama Social, 26, 93-108.

Hughes, C., Copeland, S. R., Guth, C., Rung, L. L., Hwang, B., Kleeb, G. y Strong, M. (2001). General education students' perspectives on their involvement in a Hig School peer buddy program. Education and Training in Mental Retardation and Developmental Disabilities, 36, 343-356.

Infante, M. y Gómez, V. (2004). Actitudes de los estudiantes de educación hacia la integración de personas con discapacidad y hacia la educación multicultural. Cultura y Educación, 16, 371-384. http://doi.org/10.1174/1135640042802455

Jiménez, A. y Huete, A. (2016). Informe Olivenza, 2016 sobre la situación de la discapacidad en España. Madrid: Observatorio Estatal de la Discapacidad-OED.

Krahé, B. y Altwasser, C. (2006). Changing negative attitudes towards persons with physical disabilities: an experimental intervention. Journal of Community and Applied Social Psychology, 16, 59-69. http://doi.org/10.1002/casp.849

Lagoke, B. A., Kololñafe, M. D., Ige, N. A. y Oladejo, M. A. (2010). Disability status and academic performance in a Nigerian university: Instructional implications for inclusive distance education practice. European Journal of Social Sciences, 17, 303-315.

Luque de la Rosa, A. (2010). El alumnado de la Universidad de Almería ante el colectivo de estudiantes universitarios con necesidades educativas especiales asociadas a una discapacidad. Educación y futuro digital.

Luque, A., Carrión, J. J. y Fernández, M. M. (2017). Competencias docentes para la atención a la diversidad. En J. C. Núñez, M. C. Pérez-Fuentes, M. M. Molero, J. J. Gázquez, A. B. Barragán, M. M., Simón y C. M. Hernández (Comps.), Perspectivas psicológica y educativa de las Necesidades Educativas Especiales (pp. 158-164). Almería, España: SCINFOPER.

Luque de la Rosa, A. y Gutiérrez Cáceres, R. (2013). La integración educativa y social del alumnado con discapacidad en el EEES: Universidad de Bolonia. Revista Complutense de Educación, 25, 153-175. http://doi.org/10.5209/rev_RCED.2014. v25.n1.41344

Martínez, M. A. (2010). Los estudiantes con discapacidad en la Universidad de Burgos (Tesis doctoral inédita). Burgos: Universidad de Burgos. 
McAndrew, P., Farrow, R. y Cooper, M. (2012). Adapting online learning resources for all: Planning for professionalism in accessibility. Research in Learning Technology, $20,345-361$.

Moneo, B. A. y Anaut, S. (2017). Inclusión del alumnado con discapacidad en los estudios superiores. Ideas y actitudes del colectivo estudiantil. Revista Española de Discapacidad, 5, 129-148. http://doi.org/10.5569/2340-5104.05.02.07

Moreno, F. J., Rodríguez, I. E., Saldaña, D. y Aguilera, A. (2006). Actitudes ante la discapacidad en el alumnado universitario matriculado en materias afines. Revista Iberoamericana de Educación, 40, 1-12.

Moriña, A. (2008). ¿Cómo hacer que un centro sea inclusivo? Análisis de diseño, desarrollo y resultados de un programa formativo. Revista de Investigación Educativa, 26, 521-538.

Moriña, A. (2017). Inclusive education in higher education: challengers and opportunities. European Journal of Special Needs Education, 32, 3-17.

Muñoz-Cantero, J. M., Novo-Corti, I. y Espiñeira-Bellón, E. V. (2013). La inclusión de los Estudiantes universitarios con discapacidad en las universidades presenciales: actitudes e intención de apoyo por parte de sus compañeros. Estudios sobre educación, 24, 103-124.

Nabeel, T. (2009). Teacher education for distance learning based special education in Pakistan. Turkish Online Journal of Distance Education, 10, 83-95.

Novo-Corti, I. y Muñoz-Cantero, J. M. (2012). Los estudiantes universitarios ante la inclusión de sus compañeros con discapacidad: indicadores basados en la Teoría de la Acción Razonada para los estudios de Economía y Empresa en la Universidad de A Coruña (España). Revista Española de Orientación y Pedagogía, 23, 105-122.

Richardson, J. T. E. (2010). Course completion and attainment in disabled students taking courses with the open university UK. Open Learning, 25, 81-94.

Rodríguez-Martín, A. y Álvarez-Arregui, E. (2014). Estudiantes con discapacidad en la Universidad. Un estudio sobre su inclusión. Revista Complutense de Educación, 25, 457-479. http://doi.org/10.5209/rev_RCED.2014.v25.n2.41683

Rodríguez-Martín, A. y Álvarez-Arregui, E. (2015). Universidad y discapacidad: actitudes del profesorado y de estudiantes. Perfiles educativos, 37, 86-102.

Sánchez-Palomino, A. (coord.) (2009). Integración educativa y social de los estudiantes con discapacidad en la Universidad de Almería. Almería: Universidad de Almería.

Solesvik, M. Z., Westhead, P., Kolvereid, L. y Matlay, H. (2012). Student intentions to become self-employed: The Ukranian context. Journal of Small Bussiness and Enterprise Development, 19, 441-460.

Stovall, C. y Sedlacek, W. E. (1983). Attitudes of male and female university students toward students with different physical disabilities. Journal of College Student Personnel, 24, 325-330. 
Suriá, R. (2011). Comparative analysis of student`s attitudes toward their classmates with disabilities. Electronic Journal of Research in Educational Psychology, 9, 197216.

UNESCO (2016). Educación 2030: Declaración de Incheon y Marco de Acción para la realización del Objetivo de Desarrollo Sostenible 4: Garantizar una educación inclusiva y equitativa de calidad y promover oportunidades de aprendizaje permanente para todos. Recuperado de https://unesdoc.unesco.org/ark:/48223/ pf0000245656_spa

Valenciano, G. (2009) Construyendo un concepto de educación inclusiva: Una experiencia compartida. En Sarto Martín, M. P. y Venegas Renauld, M. E., Aspectos clave de la educación inclusiva (pp. 13-24). Salamanca: INICO. Recuperado de http://sid.usal.es/idocs/F8/FDO22224/educacion-inclusiva.pdf

Verdugo Alonso, M. A. y Arias Martínez, B. (1991). Evaluación y modificación de las actitudes hacia los minusválidos. Revista de Psicología General y Aplicada, 44, 95-102. 